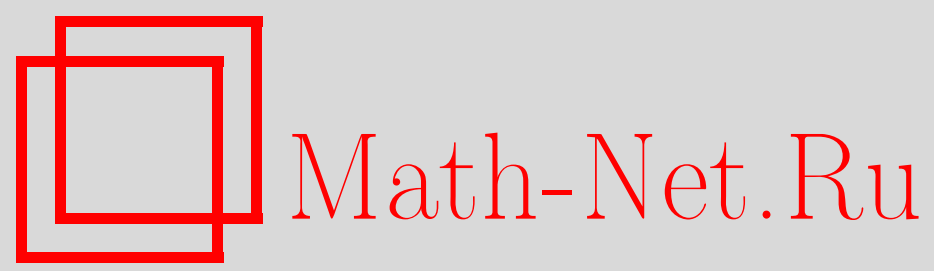

О. А. Репин, С. К. Кумыкова, Нелокальная краевая задача для системы уравнений первого порядка типа Лыкова, Вестн. Сам. гос. техн. ун-та. Сер. Физ.мат. науки, 2011, выпуск 1(), 140-150

DOI: https://doi.org/10.14498/vsgtu861

Использование Общероссийского математического портала Math-Net.Ru подразумевает, что вы прочитали и согласны с пользовательским соглашением http://www . mathnet.ru/rus/agreement

Параметры загрузки:

IP: 54.198 .67 .100

26 апреля 2023 г., 06:40:50 
УДК 517.956.32

\section{НЕЛОКАЛЬНАЯ КРАЕВАЯ ЗАДАЧА ДЛЯ СИСТЕМЫ УРАВНЕНИЙ ПЕРВОГО ПОРЯДКА ТИПА ЛЫКОВА}

\section{О.А. Репин ${ }^{1}$, С.К. Кумыкова}

1 Самарский государственный экономический университет, 443090, Самара, ул. Советской Армии, 141.

2 Кабардино-Балкарский государственный университет им. Х. М. Бербекова, 360004, Нальчик, ул. Чернышевского, 173

E-mail: matstat@mail.ru, bsk@rect.kbsu.ru

Доказана однозначная разрешимость задачи со смещением для системы дифференциальных уравнений первого порядка типа Лыкова. Доказательство проведено для различных значений параметров обобщённых операторов дробного интегро-дифференцирования, входящих в краевое условие.

Ключевые слова: нелокальная краевая задача, система дифференииальных уравнений, интегральные уравнения.

Введение. Одномерный поток влаги $u=u(\xi, t)$ в коллоидном капиллярнопористом теле поликапиллярной структуры связан с влажностью $w=w(\xi, t)$ в точке $\xi$ в момент времени $t$ обобщённым законом переноса влаги [1]

$$
u=-D \rho \frac{\partial w}{\partial \xi}-\frac{D}{a_{0}^{2}} \xi^{2} \frac{\partial u}{\partial t},
$$

где $D$ - коэффициент диффузии, $\rho$ - плотность, $a_{0}$ - коэффициент пропорциональности, зависящий от пористости тела, его капиллярных свойств и вязкости жидкости.

Если $u$ и $w$ связаны законом сохранения массы

$$
\rho \frac{\partial w}{\partial t}+\frac{\partial u}{\partial \xi}=0
$$

то получим следующую систему дифференциальных уравнений:

$$
u=-D \rho \frac{\partial w}{\partial \xi}-\frac{D}{a_{0}^{2}} \xi^{2} \frac{\partial u}{\partial t}, \quad \rho \frac{\partial w}{\partial t}+\frac{\partial u}{\partial \xi}=0 .
$$

Произведя замену $\rho w=v, x=t / a_{0}, y=\xi / a_{0}$, а затем обозначив $a=-a_{0} / D$ $(a$-безразмерная величина), системе (1) придадим следующий вид:

$$
y^{2} u_{x}+v_{y}-a u=0, \quad v_{x}+u_{y}=0 .
$$

Дифференцируя первое уравнение системы (2) по $x$, а второе по $y$ и исключив $v_{x y}$, получим уравнение А. В. Лыкова для определения $u(x, y)$ :

$$
y^{2} u_{x x}-u_{y y}-a u_{x}=0 .
$$

Олег Александрович Репин (д.ф.-м.н., профессор), зав. кафедрой, каф. математической статистики и эконометрики. Светлана Каншубиевна Кумыкова (к.ф.-м.н., доцент), доцент, каф. теории функций. 
Отметим, что уравнение (3) было приведено А. В. Бицадзе [2] как пример уравнения, для которого при $|a| \leqslant 1$ корректна по Адамару задача Коши с начальными данными на любом участке $x_{0}<x<x_{1}$ линии $y=0$ параболического вырождения, хотя нарушено известное условие Геллерстедта [3]:

$$
\lim _{y \rightarrow 0} y^{1-\frac{m}{2}} a(x, y)=0,
$$

a А. М. Нахушевым [4] - как пример уравнения, для которого при $|a|=1$ задача Дарбу поставлена некорректно и характеристики не являются равноправными носителями граничных данных.

Данная работа посвящена исследованию однозначной разрешимости нелокальной краевой задачи для системы дифференциальных уравнений Лыкова первого порядка.

1. Постановка задачи. Рассмотрим систему $(2)$ в области $D$, ограниченной характеристиками $A C: x-y^{2} / 2=0, B C: x+y^{2} / 2=1$ и отрезком $\bar{J}: 0 \leqslant x \leqslant 1$ прямой $y=0$.

ЗАДАчА. Найти решения $u(x, y), v(x, y)$ системы (2) из класса $C(\bar{D}) \cap$ $C^{1}(D \cup J)$, имеющие непрерывные вторые производные в области $D$, причём $u_{x}(x, 0), u_{y}(x, 0)$ могут иметь интегрируемые особенности при стремлении $x к$ точкам А и В, и удовлетворяющие условиям

$$
v(x, 0)=\varphi(x), \quad 0 \leqslant x \leqslant 1,
$$

$$
\begin{aligned}
A(x)\left(I_{0+}^{\delta_{1}, \delta_{2}, \frac{a-3}{4}-\delta_{1}} u\left[\theta_{0}(t)\right]\right)(x)+ & \\
+ & B(x)\left(I_{1-}^{\mu_{1}, \mu_{2},-\frac{a+3}{4}-\mu_{1}} u\left[\theta_{1}(t)\right]\right)(x)=C(x), \quad 0<x<1,
\end{aligned}
$$

где $|a|<1 ; \varphi(x), A(x), B(x), C(x)$ - известные функиии такие, что

$$
\varphi(x) \in C^{2}(\bar{J}) \cap C^{3}(J), \quad \varphi^{\prime}(0)=\varphi^{\prime}(1)=0,
$$

$A(x), B(x), C(x) \in C^{1}(\bar{J}) \cap C^{3}(J), A^{2}(x)+B^{2}(x) \neq 0 \quad \partial \Omega я \forall(x) \in[0,1] ; \delta_{1}, \delta_{2}$, $\mu_{1}, \mu_{2}$ - действительные постоянные, на которые ниже будут наложены определенные условия.

Здесь $\theta_{0}(x)$ и $\theta_{1}(x)$ - точки пересечения характеристик системы $(2)$, выходящих из точки $(x, 0)(0<x<1)$, с характеристиками $A C$ и $B C$ соответственно; $\left(I_{0+}^{\alpha, \beta, \eta} f\right)(x)$ и $\left(I_{1-}^{\alpha, \beta, \eta} f\right)(x)$ - обобщённые интегралы и производные с гипергеометрической функцией Гаусса $F(a, b ; c ; z)$, введённые в [5] (см. также $[6$, c. 326,327$])$ и имеющие при действительных $\alpha, \beta, \eta$ и $x>0$ следующий вид:

$$
\left(I_{0+}^{\alpha, \beta, \eta} f\right)(x)=\frac{x^{-\alpha-\beta}}{\Gamma(\alpha)} \int_{0}^{x}(x-t)^{\alpha-1} F\left(\alpha+\beta,-\eta ; \alpha ; 1-\frac{t}{x}\right) f(t) d t,
$$

где $0<x<1, \alpha>0$;

$$
\left(I_{0+}^{\alpha, \beta, \eta} f\right)(x)=\left(\frac{d}{d x}\right)^{n}\left(I_{0+}^{\alpha+n, \beta-n, \eta-n} f\right)(x),
$$


где $0<x<1, \alpha<0, n=[-\alpha]+1$;

$$
\left(I_{1-}^{\alpha, \beta, \eta} f\right)(x)=\frac{(1-x)^{-\alpha-\beta}}{\Gamma(\alpha)} \int_{x}^{1}(t-x)^{\alpha-1} F\left(\alpha+\beta,-\eta ; \alpha ; \frac{t-x}{1-x}\right) f(t) d t,
$$

где $0<x<1, \alpha>0$;

$$
\left(I_{1-}^{\alpha, \beta, \eta} f\right)(x)=\left(-\frac{d}{d x}\right)^{n}\left(I_{1-}^{\alpha+n, \beta-n, \eta-n} f\right)(x),
$$

где $0<x<1, \alpha<0, n=[-\alpha]+1$.

При $\beta=-\alpha$ операторы (7)-(10) сводятся к дробным интегралам и производным Римана-Лиувилля [6, с. 42,44$]$ :

$$
\left(I_{0+}^{\alpha} f\right)(x)=\left(I_{0+}^{\alpha,-\alpha, \eta} f\right)(x)=\frac{1}{\Gamma(\alpha)} \int_{0}^{x}(x-t)^{\alpha-1} f(t) d t,
$$

где $0<x<1, \alpha>0$;

$$
\left(D_{0+}^{\alpha} f\right)(x)=\left(I_{0+}^{-\alpha, \alpha, \eta} f\right)(x)=\left(\frac{d}{d x}\right)^{n}\left(I_{0+}^{n-\alpha} f\right)(x),
$$

где $0<x<1, \alpha>0, n=[\alpha]+1$;

$$
\left(I_{1-}^{\alpha} f\right)(x)=\left(I_{1-}^{\alpha,-\alpha, \eta} f\right)(x)=\frac{1}{\Gamma(\alpha)} \int_{x}^{1}(t-x)^{\alpha-1} f(t) d t,
$$

где $0<x<1, \alpha>0$;

$$
\left(D_{1-}^{\alpha} f\right)(x)=\left(I_{1-}^{-\alpha, \alpha, \eta} f\right)(x)=\left(-\frac{d}{d x}\right)^{n}\left(I_{1-}^{n-\alpha} f\right)(x),
$$

где $0<x<1, \alpha>0, n=[\alpha]+1$.

2. Однозначная разрешимость исследуемой задачи. Дифференцируя первое уравнение системы (2) по $x$, а второе - по $y$, получим

$$
\left\{\begin{array}{l}
y^{2} u_{x x}+v_{y x}-a u_{x}=0 \\
v_{x y}+u_{y y}=0
\end{array}\right.
$$

Исключая $v_{x y}$ из системы, получим уравнение А. В. Лыкова для определения $u(x, y)$ :

$$
y^{2} u_{x x}-u_{y y}-a u_{x}=0
$$

В характеристических координатах $\xi=x-y^{2} / 2, \eta=x+y^{2} / 2$ уравнение (13) преобразуется в хорошо известное уравнение Эйлера-ПуассонаДарбу (ЭПД).

Используя общее решение уравнения ЭПД [7] и возвращаясь к прежним координатам $(x, y)$, выпишем общее решение уравнения (13): 


$$
\begin{aligned}
u(x, y)=\int_{0}^{1} \Phi\left[x+\frac{y^{2}}{2}(1-2 t)\right] t^{-\frac{a+3}{4}}(1-t)^{\frac{a-3}{4}} d t- & \\
& \quad-y \int_{0}^{1} \Psi^{\prime}\left[x+\frac{y^{2}}{2}(1-2 t)\right] t^{-\frac{1+a}{4}}(1-t)^{\frac{a-1}{4}} d t .
\end{aligned}
$$

На основании (14) и второго уравнения системы $(2)\left(v_{x}=-u_{y}\right)$ имеем

$$
\begin{aligned}
v(x, y)=y \int_{0}^{1}(2 t & -1) \Phi\left[x+\frac{y^{2}}{2}(1-2 t)\right] t^{-\frac{a+3}{4}}(1-t)^{\frac{a-3}{4}} d t+ \\
+ & \int_{0}^{1} \Psi\left[x+\frac{y^{2}}{2}(1-2 t)\right] t^{-\frac{1+a}{4}}(1-t)^{\frac{a-1}{4}} d t- \\
& \quad-y^{2} \int_{0}^{1}(2 t-1) \Psi^{\prime}\left[x+\frac{y^{2}}{2}(1-2 t)\right] t^{-\frac{1+a}{4}}(1-t)^{\frac{a-1}{4}} d t .
\end{aligned}
$$

Здесь $\Phi(x)$ и $\Psi^{\prime}(x)$ - произвольные дважды непрерывно дифференцируемые функции.

Полагая в (15) $y=0$, найдём

$$
v(x, 0)=\Psi(x) \int_{0}^{1} t^{-\frac{1+a}{4}}(1-t)^{\frac{a-1}{4}} d t .
$$

Принимая во внимание условие (4) и формулу [8, п. 1.5 (1), с. 23]

$$
\int_{0}^{1} t^{\alpha-1}(1-t)^{\beta-1} d t=B(\alpha, \beta), \quad \operatorname{Re} \alpha>0, \quad \operatorname{Re} \beta>0,
$$

где $B(\alpha, \beta)$ - бета-функция, будем иметь

$$
\Psi(x)=\frac{\varphi(x)}{B\left(\frac{3-a}{4}, \frac{3+a}{4}\right)} .
$$

На основании формулы (14) и определений (7), (9) вычислим $u\left[\theta_{0}(x)\right]$ и $u\left[\theta_{1}(x)\right][9]$ :

$$
\begin{gathered}
u\left[\theta_{0}(x)\right]=\Gamma\left(\frac{1-a}{4}\right)\left(I_{0+}^{\frac{1-a}{4}, 0, \frac{a-3}{4}} \Phi(t)\right)(x)-\Gamma\left(\frac{3-a}{4}\right)\left(I_{0+}^{\frac{3-a}{4},-\frac{1}{2}, \frac{a-3}{4}} \Psi^{\prime}(t)\right)(x), \\
u\left[\theta_{1}(x)\right]=\Gamma\left(\frac{a+1}{4}\right)\left(I_{1-}^{\frac{a+1}{4}, 0,-\frac{a+3}{4}} \Phi(t)\right)(x)-\Gamma\left(\frac{a+3}{4}\right)\left(I_{1-}^{\frac{a+3}{4},-\frac{1}{2},-\frac{a+3}{4}} \Psi^{\prime}(t)\right)(x) .
\end{gathered}
$$

Подставив $u\left[\theta_{0}(x)\right]$ и $u\left[\theta_{1}(x)\right]$ в краевое условие $(5)$ и воспользовавшись формулами композиций $[6$, с. 327$]$

$$
\begin{aligned}
& \left(I_{0+}^{\alpha, \beta, \eta}\left(I_{0+}^{\gamma, \delta, \alpha+\eta} f\right)(t)\right)(x)=\left(I_{0+}^{\alpha+\gamma, \beta+\delta, \eta} f(t)\right)(x), \\
& \left(I_{1-}^{\alpha, \beta, \eta}\left(I_{1-}^{\gamma, \delta, \alpha+\eta} f\right)(t)\right)(x)=\left(I_{1-}^{\alpha+\gamma, \beta+\delta, \eta} f(t)\right)(x),
\end{aligned}
$$

где $\gamma>0$, будем иметь 


$$
\begin{aligned}
A(x) \Gamma\left(\frac{1-a}{4}\right)( & \left.I_{0+}^{\delta_{1}+\frac{1-a}{4}, \delta_{2}, \frac{a-3}{4}-\delta_{1}} \Phi(t)\right)(x)+ \\
& +B(x) \Gamma\left(\frac{1+a}{4}\right)\left(I_{1-}^{\mu_{1}+\frac{a+1}{4}, \mu_{2},-\frac{a+3}{4}-\mu_{1}} \Phi(t)\right)(x)=F(x),
\end{aligned}
$$

где

$$
\begin{aligned}
F(x)=C(x)+A(x) \Gamma\left(\frac{3-a}{4}\right)\left(I_{0+}^{\delta_{1}+\frac{3-a}{4}, \delta_{2}-\frac{1}{2}, \frac{a-3}{4}-\delta_{1}} \Psi^{\prime}(t)\right)(x)+ \\
+B(x) \Gamma\left(\frac{3+a}{4}\right)\left(I_{1-}^{\mu_{1}+\frac{a+3}{4}, \mu_{2}-\frac{1}{2},-\frac{a+3}{4}-\mu_{1}} \Psi^{\prime}(t)\right)(x) .
\end{aligned}
$$

Справедливы следующие утверждения.

Теорема 1. Если

$$
\delta_{1}=\frac{1-a}{4}, \quad \delta_{2}=0, \quad \mu_{1}+\mu_{2}=-\frac{a+1}{4}, \quad-\frac{5+a}{4}<\mu_{1}<-\frac{a+3}{4}, \quad B(x) \neq 0
$$

или

$$
\delta_{1}=\frac{1-a}{4}, \quad \delta_{2}=0, \quad \mu_{1}+\mu_{2}=-\frac{a+1}{4}, \quad-\frac{1+a}{4}<\mu_{1}<\frac{3-a}{4}, \quad A(x) \neq 0,
$$

то решение задачи (2), (4), (5) существует и единственно.

Доказ атель ств о. Пусть справедливы условия (19) теоремы 1. Тогда в силу формулы (12) уравнение (18) принимает вид

$$
\frac{\Gamma\left(\frac{1-a}{4}\right)}{\Gamma\left(\frac{1+a}{4}\right)} \frac{A(x)}{B(x)} \Phi(x)+\left(D_{1-}^{-\left(\mu_{1}+\frac{a+1}{4}\right)} \Phi(t)\right)(x)=\frac{1}{\Gamma\left(\frac{1+a}{4}\right)} \frac{F(x)}{B(x)}
$$

или

$$
\frac{\Gamma\left(\frac{1-a}{4}\right)}{\Gamma\left(\frac{1+a}{4}\right)} \frac{A(x)}{B(x)} \Phi(x)-\frac{1}{\Gamma\left(\frac{5+a}{4}+\mu_{1}\right)} \frac{d}{d x} \int_{x}^{1} \frac{\Phi(t) d t}{(t-x)^{-\frac{1+a}{4}-\mu_{1}}}=\frac{1}{\Gamma\left(\frac{1+a}{4}\right)} \frac{F(x)}{B(x)} .
$$

$\mathrm{K}$ уравнению (21) применим формулу обращения [6, с. 38,39], [10, с. 47, 48]

$$
f(x)=-\frac{\sin \pi \mu}{\pi} \frac{d}{d x} \int_{x}^{1} \frac{F(t) d t}{(t-x)^{1-\mu}}
$$

интегрального уравнения Абеля

$$
\int_{x}^{1} \frac{f(t) d t}{(t-x)^{\mu}}=F(x), \quad 0<\mu<1,
$$

и в результате получим

$$
\Phi(x)+\int_{x}^{1} K(x, t) \Phi(t) d t=F_{1}(x),
$$

где

$$
K(x, t)=\frac{\pi \Gamma\left(\frac{a+5}{4}+\mu_{1}\right) \Gamma\left(\frac{1-a}{4}\right)}{\sin \pi\left(\frac{a+5}{4}+\mu_{1}\right) \Gamma\left(\frac{1+a}{4}\right)} \frac{A(t)}{B(t)} \frac{1}{(t-x)^{\frac{a+5}{4}+\mu_{1}}},
$$




$$
F_{1}(x)=\frac{\pi \Gamma\left(\frac{a+5}{4}+\mu_{1}\right)}{\sin \pi\left(\frac{a+5}{4}+\mu_{1}\right) \Gamma\left(\frac{1+a}{4}\right)} \int_{x}^{1} \frac{F(t) d t}{B(t)(t-x)^{\frac{a+5}{4}+\mu_{1}}} .
$$

Уравнение (22) есть интегральное уравнение Вольтерра второго рода со слабой особенностью в ядре. Для исследования гладкости правой части $F_{1}(x)$ уравнения (22) преобразуем интегралы, входящие в $F(x)$.

Нетрудно видеть, что при $\delta_{1}=(1-a) / 4, \delta_{2}=0$ имеем

$$
\begin{aligned}
J_{1}(x)=\left(I_{0+}^{\delta_{1}+\frac{3-a}{4}, \delta_{2}-\frac{1}{2}, \frac{a-3}{4}-\delta_{1}} \Psi^{\prime}(t)\right)(x)=\left(I_{0+}^{1-\frac{a}{2},-\frac{1}{2}, \frac{a}{2}-1} \psi^{\prime}(t)\right)(x)= \\
=\frac{x^{\frac{a-1}{2}}}{\Gamma\left(1-\frac{a}{2}\right)} \int_{0}^{x} \Psi^{\prime}(t)(x-t)^{-\frac{a}{2}} F\left(\frac{1-a}{2}, 1-\frac{a}{2} ; 1-\frac{a}{2} ; 1-\frac{t}{x}\right) d t .
\end{aligned}
$$

Известное свойство гипергеометрической функции $F(a ; b ; b ; z)=(1-z)^{-a}$ [8, п. $2.8(4)$, с. 109] позволяет записать $J_{1}(x)$ следующим образом:

$$
\begin{aligned}
J_{1}(x)=\frac{1}{\Gamma\left(1-\frac{a}{2}\right)} \int_{0}^{x} \Psi^{\prime}(t)(x-t)^{-\frac{a}{2}} t^{\frac{a-1}{2}} d t & \\
& =\frac{\sqrt{x}}{\Gamma\left(1-\frac{a}{2}\right)} \int_{0}^{1} \Psi^{\prime}(x z) z^{\frac{a-1}{2}}(1-z)^{-\frac{a}{2}} d z .
\end{aligned}
$$

Так как $\mu_{1}+(a+3) / 4<0, \mu_{1}+\mu_{2}=-(a+1) / 4$, то

$$
\begin{aligned}
& J_{2}(x)=\left(I_{1-}^{\mu_{1}+\frac{a+3}{4}, \mu_{2}-\frac{1}{2},-\frac{a+3}{4}-\mu_{1}} \Psi^{\prime}(t)\right)(x)=\left(D_{1-}^{-\left(\mu_{1}+\frac{a+3}{4}\right)} \Psi^{\prime}(t)\right)(x)= \\
& =\frac{-1}{\Gamma\left(\frac{a+7}{4}+\mu_{1}\right)} \frac{d}{d x} \int_{x}^{1}(t-x)^{\frac{a+3}{4}+\mu_{1}} \Psi^{\prime}(t) d t= \\
& =\frac{-(1-x)^{\frac{a+7}{4}+\mu_{1}}}{\Gamma\left(\frac{a+7}{4}+\mu_{1}\right)} \int_{0}^{1} \Psi^{\prime \prime}(1-(1-x) z)(1-z)^{\frac{a+3}{4}+\mu_{1}} d z .
\end{aligned}
$$

Соотношения (23), $(24)$ и условия (6) позволяют утверждать, что $F(x) \in$ $C([0,1]) \cap C^{1}(J)$. Отсюда заключаем, что $F_{1}(x) \in C(\bar{J}) \cap C^{2}(J)$.

Таким образом, уравнение (22) есть интегральное уравнение Вольтерра второго рода при $B(x) \neq 0$ со слабой особенностью в ядре и правой частью $F_{1}(x) \in C(\bar{J}) \cap C^{2}(J)$. В этом классе функций уравнение $(22)$ имеет единственное решение $\Phi(x)$, которое может быть построено методом последовательных приближений [10].

По найденным $\Phi(x)$ и $\Psi(x)$ решения $u(x, y), v(x, y)$ задачи $(4),(5)$ для системы (2) находятся по формулам (14), (15).

При выполнении условий (20) теоремы 1 уравнение (22) принимает вид

$$
\Phi(x)+\int_{x}^{1} K_{1}(x, t) \Phi(t) d t=F_{2}(x)
$$

где

$$
K_{1}(x, t)=\frac{\Gamma\left(\frac{1+a}{4}\right)}{\Gamma\left(\frac{1-a}{4}\right)} \frac{B(x)}{A(x)} \frac{1}{(t-x)^{\frac{3-a}{4}-\mu_{1}}}, \quad F_{2}(x)=\frac{1}{\Gamma\left(\frac{1-a}{4}\right) A(x)} F(x) .
$$


Уравнение (25) также является интегральным уравнением Вольтерра второго рода при $A(x) \neq 0$ со слабой особенностью в ядре $K_{1}(x, t)$ и правой частью $F_{2}(x) \in C(\bar{J}) \cap C^{1}(J)$.

Далее аргументация доказательства теоремы 1 аналогична.

Tеорема 2. Eсли $\mu_{1}=-(a+1) / 4, \mu_{2}=0, \delta_{1}+\delta_{2}=(a-1) / 4,(a-5) / 4<$ $<\delta_{1}<(a-1) / 4, A(x) \neq 0$ или $\mu_{1}=-(a+1) / 4, \mu_{2}=0, \delta_{1}+\delta_{2}=(a-1) / 4$, $(a-1) / 4<\delta_{1}<(a+3) / 4, B(x) \neq 0$, то решение задачи (2), (4), (5) существует и единственно.

Доказ ательств в теоремы 2 аналогично доказательству теоремы 1.

Teоpema 3. Если $(a+1) / 4<\delta_{1}<(a+3) / 4, \delta_{2}=-1, \mu_{1}=\delta_{1}-a / 2$, $\mu_{2}=-\left(\delta_{1}+(1-a) / 4\right), A(x)=A=$ const $\neq 0, B(x)=B=$ const $\neq 0 u$ выполняются условия (6), то решение задачи (2), (4), (5) существует.

Доказательство. При выполнении условий теоремы 3 соотношение (18) принимает вид

$$
\begin{aligned}
A \Gamma\left(\frac{1-a}{4}\right)\left(I_{0+}^{\delta_{1}+\frac{1-a}{4},-1, \frac{a-3}{4}-\delta_{1}} \Phi(t)\right)(x)+ & \\
& +B \Gamma\left(\frac{1+a}{4}\right)\left(I_{1-}^{\delta_{1}+\frac{1-a}{4},-\left(\delta_{1}+\frac{1-a}{4}\right), \frac{a-3}{4}-\delta_{1}} \Phi(t)\right)(x)=F_{3}(x),
\end{aligned}
$$

где

$$
\begin{aligned}
F_{3}(x)=C(x)+A \Gamma\left(\frac{3-a}{4}\right) & \left(I_{0+}^{\delta_{1}+\frac{3-a}{4},-\frac{3}{2}, \frac{a-3}{4}-\delta_{1}} \Psi^{\prime}(t)\right)(x)+ \\
+ & B \Gamma\left(\frac{3+a}{4}\right)\left(I_{1-}^{\delta_{1}+\frac{3-a}{4}, \frac{a-3}{4}-\delta_{1},-\delta_{1}-\frac{3-a}{4}} \Psi^{\prime}(t)\right)(x) .
\end{aligned}
$$

Применив к обеим частям (26) оператор $I_{0+}^{-\left(\delta_{1}+\frac{1-a}{4}\right), 1,-\frac{1}{2}}$ и используя первую формулу композиций из (17), будем иметь

$$
\begin{aligned}
A \Gamma\left(\frac{1-a}{4}\right) & \Phi(x)+B \Gamma\left(\frac{1+a}{4}\right) \times \\
& \times\left(I_{0+}^{-\left(\delta_{1}+\frac{1-a}{4}\right), 1,-\frac{1}{2}} I_{1-}^{\delta_{1}+\frac{1-a}{4},-\left(\delta_{1}+\frac{1-a}{4}\right), \frac{a-3}{4}-\delta_{1}} \Phi(t)\right)(x)= \\
& =\left(I_{0+}^{-\left(\delta_{1}+\frac{1-a}{4}\right), 1,-\frac{1}{2}} F_{3}(t)\right)(x) .
\end{aligned}
$$

В работе [11, с. 349] получена формула

$$
I_{0+}^{-p, 1, r}\left(I_{1-}^{p,-p, s} f(t)\right)(x)=x^{p-1} \cos \pi p f(x)+\int_{0}^{1} K(x, u) f(u) d u,
$$

где $0<p<1$ и

$$
K(x, u)= \begin{cases}\frac{-\Gamma(r+1)}{\Gamma(1-p) \Gamma(p+r)} x^{-r-1} u^{p+r-1} F\left(p ; r+1 ; r+p ; \frac{u}{x}\right), & 0<u<x ; \\ \frac{-\Gamma(r+1)}{\Gamma(p-1) \Gamma(-p+r+2)} u^{p-2} F\left(r+1 ; 2-p ;-p+r+2 ; \frac{x}{u}\right), & x<u<1 .\end{cases}
$$

146 
Используя (28), полагая $p=\delta_{1}+(1-a) / 4, r=-1 / 2, s=(a-3) / 4$ и учитывая, что $\Gamma(1 / 2)=\sqrt{\pi}[8$, п. $1.2(10)$, с. 18], перепишем $(27)$ в виде интегрального уравнения

$$
\begin{aligned}
& {\left[A \Gamma\left(\frac{1-a}{4}\right)+B \Gamma\left(\frac{1+a}{4}\right) \cos \pi\left(\delta_{1}+\frac{1-a}{4}\right) x^{\delta_{1}-\frac{a+3}{4}}\right] \Phi(x)+} \\
& +\int_{0}^{x} K_{11}(x, t) \Phi(t) d t+\int_{x}^{1} K_{12}(x, t) \Phi(t) d t=\left(I_{0+}^{-\left(\delta_{1}+\frac{1-a}{4}\right), 1,-\frac{1}{2}} F_{3}(t)\right)(x),
\end{aligned}
$$

где

$$
\begin{aligned}
& K_{11}(x, t)=\frac{-\sqrt{\pi}}{\Gamma\left(\frac{a+3}{4}-\delta_{1}\right) \Gamma\left(\delta_{1}-\frac{a+1}{4}\right)} x^{-\frac{1}{2}} t^{\delta_{1}-\frac{a+5}{4}} \times \\
& \quad \times F\left(\delta_{1}+\frac{1-a}{4}, \frac{1}{2} ; \delta_{1}-\frac{a+1}{4} ; \frac{t}{x}\right), \quad 0<t<x ; \\
& K_{12}(x, t)=\frac{-\sqrt{\pi}}{\Gamma\left(\delta_{1}-\frac{a+3}{4}\right) \Gamma\left(\frac{5+a}{4}-\delta_{1}\right)} t^{\delta_{1}-\frac{a+7}{4} \times} \\
& \quad \times F\left(\frac{1}{2}, \frac{a+7}{4}-\delta_{1} ; \frac{a+5}{4}-\delta_{1} ; \frac{x}{t}\right), \quad x<t<1 .
\end{aligned}
$$

Таким образом, существование решения задачи редуцировано к вопросу разрешимости уравнения (29). Исследуем гладкость его правой части. Найдем композиции обобщённых операторов правой части уравнения (29). Воспользуемся вначале первой формулой из (17):

$$
\begin{aligned}
& I_{3}(x)=I_{0+}^{-\left(\delta_{1}+\frac{1-a}{4}\right), 1,-\frac{1}{2}}\left(I_{0+}^{\delta_{1}+\frac{3-a}{4},-\frac{3}{2}, \frac{a-3}{4}-\delta_{1}} \Psi^{\prime}(t)\right)(x)= \\
& =\left(I_{0+}^{\frac{1}{2},-\frac{1}{2},-\frac{1}{2}} \Psi^{\prime}(t)\right)(x)=\left(I_{0+}^{\frac{1}{2}} \Psi^{\prime}(t)\right)(x) .
\end{aligned}
$$

Учитывая определение (11) и равенство $\Psi^{\prime}(0)=0$, после интегрирования по частям можно записать

$$
I_{3}(x)=\frac{1}{\sqrt{\pi}} \int_{0}^{x}(x-t)^{-\frac{1}{2}} \Psi^{\prime}(t) d t=\sqrt{\frac{x}{\pi}} \int_{0}^{1} \frac{\Psi^{\prime}(x z) d z}{\sqrt{1-z}} .
$$

Откуда следует, что $I_{3}(x) \in C(\bar{J}) \cap C^{2}(J)$.

Далее рассмотрим

$$
\begin{gathered}
I_{4}(x)=I_{0+}^{-\left(\delta_{1}+\frac{1-a}{4}\right), 1,-\frac{1}{2}}\left(I_{1-}^{\delta_{1}+\frac{1-a}{4},-\left(\delta_{1}+\frac{1-a}{4}\right), \frac{a-3}{4}-\delta_{1}} \Psi^{\prime}(t)\right)(x)= \\
=\frac{d}{d x}\left(I_{0+}^{\frac{a+3}{4}-\delta_{1}, 0,-\frac{3}{2}}\left(I_{1-}^{\delta_{1}+\frac{3-a}{4}} \Psi^{\prime}(t)\right)(s)\right)(x)= \\
=\frac{d}{d x} \frac{x^{\delta_{1}-\frac{a+3}{4}}}{\Gamma\left(\frac{a+3}{4}-\delta_{1}\right)} \int_{0}^{x}(x-t)^{\frac{a-1}{4}-\delta_{1}} F\left(\frac{a+3}{4}-\delta_{1}, \frac{3}{2} ; \frac{a+3}{4}-\delta_{1} ; 1-\frac{t}{x}\right) d t \times
\end{gathered}
$$




$$
\times \frac{1}{\Gamma\left(\delta_{1}+\frac{3-a}{4}\right)} \int_{t}^{1}(s-t)^{\delta_{1}-\frac{a+1}{4}} \Psi^{\prime}(s) d s .
$$

Поменяв порядок интегрирования, с учётом формулы

$$
\int_{0}^{x} d t \int_{t}^{1} d s=\int_{0}^{x} d s \int_{0}^{s} d t+\int_{x}^{1} d s \int_{0}^{x} d t
$$

и свойства гипергеометрической функции Гаусса $F(a, b ; a ; z)=(1-z)^{-b}$ получим

$$
\begin{aligned}
& I_{4}(x)=\frac{1}{\Gamma\left(\frac{a+3}{4}-\delta_{1}\right) \Gamma\left(\delta_{1}+\frac{3-a}{4}\right)} \frac{d}{d x} x^{\delta_{1}-\frac{a+3}{4}} \times \\
& \times\left[\int_{0}^{x} \Psi^{\prime}(s) d s \int_{0}^{s} t^{-\frac{3}{2}}(x-t)^{\frac{a-1}{4}-\delta_{1}}(s-t)^{\delta_{1}-\frac{a+1}{4}} d t+\right. \\
& \left.+\int_{x}^{1} \Psi^{\prime}(s) d s \int_{0}^{x} t^{-\frac{3}{2}}(x-t)^{\frac{a-1}{4}-\delta_{1}}(s-t)^{\delta_{1}-\frac{a+1}{4}} d t\right]= \\
& =\frac{1}{\Gamma\left(\frac{a+3}{4}-\delta_{1}\right) \Gamma\left(\delta_{1}+\frac{3-a}{4}\right)} \frac{d}{d x} x^{\delta_{1}-\frac{a+3}{4}}\left[\frac{\Gamma\left(-\frac{1}{2}\right) \Gamma\left(\delta_{1}+\frac{3-a}{4}\right)}{\Gamma\left(\delta_{1}+\frac{1-a}{4}\right)} \times\right. \\
& \times \int_{0}^{x} s^{\delta_{1}-\frac{a+3}{4}} x^{\frac{a-1}{4}-\delta_{1}} F\left(-\frac{1}{2}, \delta_{1}-\frac{a-1}{4} ; \delta_{1}-\frac{a-1}{4} ; \frac{s}{x}\right) \Psi^{\prime}(s) d s+ \\
& +\frac{\Gamma\left(-\frac{1}{2}\right) \Gamma\left(\frac{a+3}{4}-\delta_{1}\right)}{\Gamma\left(\frac{a+1}{4}-\delta_{1}\right)} \times \\
& \left.\times \int_{x}^{1} s^{\delta_{1}-\frac{a+1}{4}} x^{\frac{a-3}{4}-\delta_{1}} F\left(-\frac{1}{2}, \frac{a+1}{4}-\delta_{1} ; \frac{a+1}{4}-\delta_{1} ; \frac{x}{s}\right) \Psi^{\prime}(s) d s\right] .
\end{aligned}
$$

В силу формулы $\Gamma(-1 / 2)=-2 \sqrt{\pi}[12$, п. II.1, с. 773] окончательно имеем

$$
\begin{aligned}
& I_{4}(x)=-\frac{2 \sqrt{\pi}}{\Gamma\left(\frac{a+3}{4}-\delta_{1}\right) \Gamma\left(\delta_{1}+\frac{1-a}{4}\right)} \frac{d}{d x} \int_{0}^{x} s^{\delta_{1}-\frac{a+3}{4}}(x-s)^{\frac{1}{2}} \Psi^{\prime}(s) d s- \\
& -\frac{2 \sqrt{\pi}}{\Gamma\left(\delta_{1}+\frac{3-a}{4}\right) \Gamma\left(\frac{a+1}{4}-\delta_{1}\right)} \frac{d}{d x} \int_{x}^{1} s^{\delta_{1}-\frac{a+3}{4}}(s-x)^{\frac{1}{2}} \Psi^{\prime}(s) d s .
\end{aligned}
$$

Дифференцируя по $x$, производя замену в первом интеграле $s=x z$, а во втором $-s=1-(1-x) z$ и учитывая формулу $\Gamma(z) \Gamma(1-z)=\pi / \sin \pi z[8$, п. 1.2 (6), с. 18], получим

$$
\begin{gathered}
I_{4}(x)=-\frac{1}{\sqrt{\pi}}\left[\sin \pi\left(\frac{3+a}{4}-\delta_{1}\right) x^{\delta_{1}-\frac{a+1}{4}} \int_{0}^{1}(1-z)^{-\frac{1}{2}} z^{\delta_{1}-\frac{a+3}{4}} \Psi^{\prime}(x z) d z-\right. \\
\left.-\sin \pi\left(\frac{3-a}{4}+\delta_{1}\right)(1-x)^{\frac{1}{2}} \int_{0}^{1}(1-z)^{-\frac{1}{2}}(1-(1-x) z)^{\delta_{1}-\frac{a+3}{4}} \Psi^{\prime}(1-(1-x) z) d z\right] .
\end{gathered}
$$

Отсюда следует, что $I_{4}(x) \in C(\bar{J}) \cap C^{2}(J)$. 
Теперь преобразуем первое слагаемое в правой части интегрального уравнения (29):

$$
\begin{gathered}
I_{5}(x)=\left(I_{0+}^{-\left(\delta_{1}+\frac{1-a}{4}\right), 1,-\frac{1}{2}} C(t)\right)(x)=\frac{d}{d x}\left(I_{0+}^{\frac{a+3}{4}-\delta_{1}, 0,-\frac{3}{2}} C(t)\right)(x)= \\
=\frac{1}{\Gamma\left(\frac{a+3}{4}-\delta_{1}\right)} \frac{d}{d x}\left[x^{\delta_{1}-\frac{a-3}{4}} \int_{0}^{x}(x-t)^{\frac{a-1}{4}-\delta_{1}} t^{-\frac{3}{2}} C(t) d t\right]= \\
=\frac{1}{\Gamma\left(\frac{a+3}{4}-\delta_{1}\right)} \int_{0}^{1}(1-z)^{\frac{a-1}{4}-\delta_{1}} z^{-\frac{1}{2}} C^{\prime}(x z) d z .
\end{gathered}
$$

Откуда следует, что $I_{5}(x) \in C(\bar{J}) \cap C^{2}(J)$.

Из явного вида ядер $K_{11}(x, t)$ и $K_{12}(x, t)$, а также свойств слагаемых $I_{3}(x), I_{4}(x), I_{5}(x)$ правой части уравнения (29) при

$$
A \Gamma\left(\frac{1-a}{4}\right)+B \Gamma\left(\frac{1+a}{4}\right) \cos \pi\left(\delta_{1}+\frac{1-a}{4}\right) x^{\delta_{1}-\frac{a+3}{4}} \neq 0
$$

следует, что уравнение (29) есть интегральное уравнение Фредгольма второго рода со слабой особенностью в ядре относительно $\Phi(x)$, где правая часть принадлежит классу $C[0,1] \cap C^{2}(0,1)$. В этом классе существует решение $\Phi(x)$ уравнения (29). Зная $\Phi(x)$ и $\Psi(x)$, используя формулы $(14),(15)$, нетрудно найти решения $u(x, y)$ и $v(x, y)$ задачи $(4),(5)$ для системы $(2)$. Доказательство теоремы 3 закончено.

\section{БИБЛИОГРАФИЧЕСКИЙ СПИСОК}

1. Лыков А.В. Применение методов термодинамики необратимых процессов к исследованию тепло и массообмена // Инж.-физ. ж., 1965. Т. 9, № 3. С. 287-304. [Lykov A. V. Application of methods of thermodynamics of irreversible process to heat and mass transfer problems // Inzh.-fiz. Zh., 1965. Vol. 9, no. 3. Pp. 287-304].

2. Бицадзе A. В. Некоторые классы уравнений в частных производных. М.: Наука, 1981. 448 c. [Bitsadze A. V. Some classes of partial differential equations. Moscow: Nauka, 1981. 448 pp.]

3. Gellerstedt $S$. Sur une équation linéaire aux dérivées partielles de type mixte// Ark. Mat. Astron. Fys. A, 1937. Vol. 25, no. 29. Pp. 1-23.

4. Нахушев А. М. О задаче Дарбу для гиперболических уравнений // ДАН CCCP, 1970. Т. 195, № 4. С. 776-779; англ. пер.: Nakhushev A. M. On the Darboux problem for hyperbolic equations // Sov. Math., Dokl., 1970. Vol. 195, no. 4. Pp. 1567-1570.

5. Saigo M. A remark on integral operators involving the Gauss hypergeometric functions // Math. Rep. Kyushu Univ., 1978. Vol.11, no. 2. Pp. 135-143.

6. Самко С.Г., Килбас А.А., Маричев О.И. Интегралы и производные дробного порядка и некоторые их приложения. Минск: Наука и техника, 1987. 688 с. [Samko S. G., Kilbas A.A., Marichev O.I. Integrals and derivatives of fractional order and some of their applications. Minsk: Nauka i Tekhnika, 1987. 688 pp.]

7. Смирнов M. M. Вырождающиеся гиперболические уравнения. Минск: Высш. шк., 1977. 160 c. [Smirnov M. M. Degenerate hyperbolic equations. Minsk: Vyssh. shk., 1977. 160 pp.]

8. Бейтмен Г., Эрдейи А. Высшие трансцендентные функции. В 3-х т. Т. 1: Гипергеометрическая функция. Функция Лежандра. М.: Наука, 1973. 296 с.; ориг.: Erdélyi A., Magnus W., Oberhettinger F., Tricomi F. G. Higher transcendental functions. Vol. I/ ed. H. Bateman. New York - Toronto - London: McGraw-Hill Book Co, Inc., 1953. 302 pp. 
9. Репин О. А. О разрешимости задачи с краевым условием на характеристиках для вырождающегося гиперболического уравнения // Дифферени. уравнения, 1998. Т. 34, № 1 . C. 110-113; англ. пер.: Repin O.A. Solvability of a problem with boundary conditions on characteristics for a degenerate hyperbolic equation // Differ. Equations, 1998. Vol.34, no. 1. Pp. 113-116.

10. Михлин С. Г. Лекции по линейным интегральным уравнениям. М.: Физматлит, 1959. 232 c. [Mihlin S. G. Lectures on linear integral equations. Moscow: Fizmatlit, 1959. 232 pp.]

11. Srivastava N. M., Saigo M. Multiplication of fractional calculus operators and boundary value problems involving the Euler-Darboux equation// J. Math. Anal. Appl., 1987. Vol. 121, no. 2. Pp. 325-369.

12. Прудников А.П., Брычков Ю.А., Маричев О.И. Интегралы и ряды. Элементарные функции. М.: Наука, 1981. 799 с. [Prudnikov A.P., Brychkov Yu.A., Marichev O.I. Integrals and series. Elementary functions. Moskva: Nauka, 1981. 799 pp.]

Поступила в редакцию 20/XII/2010;

в окончательном варианте - 26/II/2011.

MSC: 35L50; 35Q05, 47G20, 34B10

NONLOCAL BOUNDARY VALUE PROBLEM FOR A LYKOV'S TYPE SYSTEM OF FIRST-ORDER

\section{O.A. Repin ${ }^{1}$, S. K. Kumykova ${ }^{2}$}

1 Samara State Economic University,

141, Soviet Army st., Samara, 443090, Russia.

2 Kabardino-Balkarian State University,

173, Chernyshevskogo st., Nal'chik, 360004, Russia.

E-mail: matstat@mail.ru, bsk@rect.kbsu.ru

In this paper we prove the unique solution of the problem with a shift to a Lykov's type system of differential equations of first order. The proof is given for different values of the generalized operators of fractional integro-differentiation included in the boundary condition.

Key words: nonlocal value boundary problem, system of differential equations, integral equations.

Original article submitted 20/XII/2010; revision submitted $26 / \mathrm{II} / 2011$.

Oleg A. Repin (Dr. Sci. (Phys. \& Math.)), Head of Dept., Dept. of Mathematical Statistics \& Econometrics. Svetlana K. Kumykova (Ph. D. (Phys. \& Math.)), Associate Professor, Dept. of Function Theory. 